INPLASY

PROTOCOL

To cite: Niu et al. Application effect of different catheterization methods in patients with neurogenic bladder: A network metaanalysis. Inplasy protocol 202220042. doi:

10.37766/inplasy2022.2.0042

Received: 13 February 2022

Published: 13 February 2022

Corresponding author:

Tian Jinhui

tjh996@163.com

Author Affiliation:

Evidence-Based Nursing

Center, School of Nursing,

Lanzhou University.

Support: 2020-RC-63.

Review Stage at time of this submission: Formal screening of search results against eligibility criteria.

\section{Application effect of different catheterization methods in patients with neurogenic bladder: A network meta-analysis}

Niu, M1; Gao, Y2; Yan, M3; Zhang, L4; Zhang, Y5; Yu, H6; Yang, X7; Sun, J8; Tian, J9.

Review question / Objective: Comparison of the efficacy and safety of different catheterization methods in patients with neurogenic bladder using a network meta-analysis.

Condition being studied: Reasonable and effective catheterization is essential for improving and restoring bladder function in patients with neurogenic bladder and reducing the occurrence of urinary system complications. However, the existing studies and guidelines are inconclusive about the efficacy and safety of different catheterization methods in patients with neurogenic bladder.

Information sources: We will identify relevant trials from systematic searches in the following electronic databases: PubMed, EMBASE, Web of Science, Cochrane Library, CNKI, WanFang Data, China Biology Medicine disc. We will also search Clinical Trials. gov, the WHO International Clinical Trials Registry Platform for unpublished data.

INPLASY registration number: This protocol was registered with the International Platform of Registered Systematic Review and Meta-Analysis Protocols (INPLASY) on 13 February 2022 and was last updated on 13 February 2022 (registration number INPLASY202220042).

Conflicts of interest:

None declared.

\section{INTRODUCTION}

Review question / Objective: Comparison of the efficacy and safety of different catheterization methods in patients with neurogenic bladder using a network metaanalysis.
Condition being studied: Reasonable and effective catheterization is essential for improving and restoring bladder function in patients with neurogenic bladder and reducing the occurrence of urinary system complications. However, the existing studies and guidelines are inconclusive 
about the efficacy and safety of different catheterization methods in patients with neurogenic bladder.

\section{METHODS}

Participant or population: Neurogenic bladder patients.

Intervention: Comparison of different catheterization methods such as sterile intermittent catheterization, clean intermittent catheterization, indwelling catheterization, and non-contact intermittent catheterization.

Comparator: Comparison of different catheterization methods such as sterile intermittent catheterization, clean intermittent catheterization, indwelling catheterization, and non-contact intermittent catheterization.

Study designs to be included: Any relevant randomized controlled trials (RCTs) will be included. randomized controlled trial.

Eligibility criteria: The eligibility criteria will be the followingEligibility criteria are as follows: 1. Type of study: Randomised controlled trial (RCT). 2. Participants: Patients diagnosed with neurogenic bladder. 3. Interventions: sterile intermittent catheterization, clean intermittent catheterization, indwelling catheterization, non-contact intermittent catheterization.

Information sources: We will identify relevant trials from systematic searches in the following electronic databases: PubMed, EMBASE, Web of Science, Cochrane Library, CNKI, WanFang Data, China Biology Medicine disc. We will also search Clinical Trials. gov, the WHO International Clinical Trials Registry Platform for unpublished data.

Main outcome(s): Complication rate: urinary tract infection, urinary tract stones, etc.; bladder function indicators: residual urine volume, bladder pressure, bladder capacity.
Quality assessment / Risk of bias analysis: According to Cochrane Collaboration's Risk of bias tool, We will assess risk of bias as 'low risk', 'unclear risk' or 'high risk'. The following items will be evaluated: sequence generation, allocation concealment, blinding of participants and personnel, blinding of outcome assessors, incomplete outcome data, selective outcome reporting and other sources of bias (eg, sponsorship bias/researcher allegiance bias). The evaluation will be conducted by two independent raters. Any disagreements will be resolved by a third review author.

Strategy of data synthesis: We will use Stata V.15.0. software to map network plot to compare the effects of interventions. We will use relative risk (RR) for the dichotomous data, use standard mean differences (SMD) for the continuous outcome and calculate the $95 \% \mathrm{Cl}$.

Subgroup analysis: If statistical heterogeneity is evident, we will analyze the causes of heterogeneity, if there is enough data.

Sensitivity analysis: We will use the exclusion method to conduct sensitivity analysis: (1) exclude low-quality studies; (2) exclude trials with missing data.

Country(ies) involved: China.

Keywords: Neurogenic bladder; Catheterization; Randomized controlled trial; Network meta-analysis.

Contributions of each author:

Author 1 - Mingming Niu.

Email: niumm19@lzu.edu.cn

Author 2 - Ya Gao.

Author 3 - Meili Yan.

Author 4 - Li Zhang.

Author 5 - Yan Zhang.

Author 6 - Huijin Yu.

Author 7 - Xiaoxia Yang.

Author 8 - Jundi Sun.

Author 9 - Jinhui Tian.

Email: tjh996@163.com 